\title{
Chronic otorrhoea: Spectrum of microorganisms and antibiotic sensitivity in a South African cohort
}

\author{
E Meyer, ${ }^{1} \mathrm{MB}$ ChB, FCORL (SA); A Whitelaw, ${ }^{2,3} \mathrm{MB}$ ChB, MSc, FCPath (SA) (Micro); O Edkins, ${ }^{1}$ MB BCh, FCORL (SA), MMed (ORL); \\ J J Fagan, ${ }^{1}$ MB ChB, MMed, FCS (ORL)
}

'Division of Otolaryngology, University of Cape Town, South Africa

${ }^{2}$ Division of Medical Microbiology, University of Cape Town, South Africa

${ }^{3}$ National Health Laboratory Service, Groote Schuur Hospital, Cape Town, South Africa

Corresponding author: E Meyer (meyerestie@mweb.co.za)

Background. Chronic otorrhoea is difficult to treat, with treatment in South Africa (SA) being protocol driven and generally initiated at the primary healthcare level. There is a lack of local studies that focus on the bacteriology and antimicrobial sensitivities of chronic otorrhoea, which underpins the management advice offered.

Aims. To determine the microbiological profile and antimicrobial susceptibility of patients with chronic otorrhoea and the validity of the Department of Health's (DoH) current guideline.

Methods. We conducted a prospective study at Groote Schuur Hospital from 2005 to 2009. We included patients with chronic otorrhoea classified as either otitis media or otitis externa, according to our definitions. Pus swabs were taken, from which microorganisms were cultured and tested for antimicrobial susceptibility.

Results. Of 79 patients with otorrhoea, 50 had otitis media, 21 had otitis externa and the condition was not determined in 8 patients. The most common organism isolated with otitis media was Proteus mirabilis (18/50;36\%) and with otitis externa, Pseudomonas aeruginosa (7/21;33\%). Otorrhoea had a different microbial spectrum compared with international reports, with methicillin-resistant Staphylococcus aureus infection in a single patient. The organisms isolated were susceptible mainly to fluoroquinolones (96\%) and aminoglycosides (81\%).

Conclusion. Amoxicillin is a poor choice of antibiotic due to its low sensitivity, which calls into question the current DoH guideline for otorrhoea. Antimicrobial treatment protocols should be based on local data and be revisited from time to time. This study suggests that, should first-line treatment fail, an antibiotic with Gram-negative cover, e.g. a topical fluoroquinolone, should be considered.

S Afr Med J 2013;103(7):471-473. DOI:10.7196/SAMJ.6066

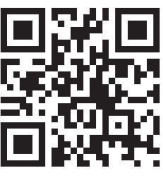

Otorrhoea due to either external or middle-ear pathology is often difficult to treat. Different geographical areas may have unique patterns of infecting organisms and antimicrobial susceptibility. Worldwide, there has been a change in the profile of organisms causing otorrhoea and increases have been observed both in the incidence of methicillin-resistant Staphylococcus aureus (MRSA) and in resistance to antimicrobials. ${ }^{[1]}$ In South Africa (SA), patients with otorrhoea are often initially treated at the primary healthcare level. Otorrhoea is seldom investigated by microscopy, culturing or sensitivity testing; treatment is usually empirical and has limited options. Although treatment is protocol driven, a PubMed search failed to reveal SA studies focusing purely on the bacteriology and antimicrobial sensitivities of chronic otorrhoea underpinning the management advice offered.

\section{Aims}

To investigate the microbiology profiles of patients presenting to a tertiary hospital in Cape Town with chronic otorrhoea and to ascertain the validity of the current treatment protocol for chronic otorrhoea outlined by the SA Department of Health (DoH).

\section{Methods}

We conducted a prospective study at the tertiary-level Groote Schuur Hospital (GSH) in Cape Town over 4 years from 2005 to 2009. Patients with chronically discharging ears, who were referred to the Ear, Nose and Throat (ENT) Outpatients Unit by primary and secondary level services, were enrolled at their first visit to the hospital.

To best reflect the clinical situation at primary or secondary care level, we defined chronic otorrhoea as an ear discharge present for $\geq 2$ weeks, either due to otitis media or otitis externa. We based the diagnosis of otitis media on the presence of a tympanic membrane (TM) perforation with the otorrhoea originating from the middle ear. We defined otitis externa as an intact TM and an inflamed, discharging lining of the external auditory canal. Our exclusion criteria included: patients with cholesteatoma, patients who had previous surgery, anatomical factors predisposing patients to recurrent ear infection, patients with foreign bodies, cerebrospinal fluid otorrhoea and patients who had received systemic or topical antibiotics in the 2 weeks prior to presenting to the hospital.

To obtain a representative sample of the ear discharge, ear toilet was first performed with a diagnostic microscope before a pus swab was carefully taken with a single mini-tip culture swab under direct microscopic vision. The swab was generally too big to pass through a TM perforation, and thus the discharge was sampled just lateral to the TM.

Pus swabs were analysed by GSH's microbiology laboratory, a SA National Accreditation System accredited laboratory, within the National Health Laboratory Service (NHLS). Swabs were inoculated onto blood agar, boiled blood agar and MacConkey agar and incubated for up to 48 hours. Isolates were identified using standard laboratory techniques and antimicrobial susceptibility testing was performed using either the Vitek 2 system (bioMérieux, France) or standard Kirby Bauer disc diffusion test. Results were interpreted using Clinical and Laboratory Standards Institute (CLSI) guidelines. ${ }^{[2]}$

\section{Results}

Seventy-nine patients met the inclusion criteria for the study with a mean age of 39 years (range $13-83$ years). The female-to-male ratio 
was 1.3:1 and the ratio of left to right ears was 1.2:1. Eight patients volunteered that they were HIV positive, 42 that they were negative and 35 did not know their HIV status. The duration of otorrhoea ranged from 4 to $>100$ weeks (mean 47 weeks). Otorrhoea had been present for $>52$ weeks in $34(43 \%)$ patients.

Of the 79 patients enrolled in the study, 50 had otitis media, 21 had otitis externa and in 8 patients this distinction was not documented. In 31 patients the treatment that they had received prior to referral had been documented and included oral antibiotics, i.e. amoxicillin $(n=24)$, ciprofloxacin $(n=2)$, flucloxacillin $(n=1)$ and amoxicillin-clavulanate $(n=1)$. Five patients had received topical acetic acid drops, 2 of whom had also received amoxicillin.

Proteus mirabilis $(36 \% ; 18 / 50)$ was the most common isolate in otitis media while Pseudomonas aeruginosa (33\%; 7/21) occurred most commonly in otitis externa (Table 1).

The patterns of susceptibility are listed in Table 2. MRSA, which was sensitive to erythromycin, was detected in only 1 patient (with otitis media) - a 35-year-old HIVpositive patient with a 2-year history of otorrhoea.

\section{Discussion}

Our results describe the microbiology associated with chronic otorrhoea (both otitis externa and otitis media) in patients referred from primary and secondary healthcare facilities in the Western Cape. The most common organisms were P. mirabilis (29\%), P. aeruginosa (20\%) and S. aureus (15\%). The microbiology associated with otitis media differed from otitis externa, with $P$. mirabilis (36\%) and P. aeruginosa (33\%) being the most common isolates, respectively (Table 1). Our results roughly parallel the microbiology pattern reported by Loock $^{[3]}$ at another tertiary hospital in Cape Town in chronic otitis media patients, namely Proteus spp. (29\%), P. aeruginosa (24\%) and S. aureus (14\%) as the most common.

A problem when comparing our results with those of some other studies is that they refer to 'chronic suppurative otitis media, defined as 'inflammation of the middle ear and mastoid process, accompanied by a perforated tympanic membrane and discharge. ${ }^{\left[{ }^{[1]}\right.}$ In such studies, sampling would include otorrhoea due to cholesteatoma (treatment of which is surgical) and non-cholesteatomatous ears. Although most studies indicate that only noncholesteatoma cases were included, this is not

\begin{tabular}{|c|c|c|c|}
\hline & $\begin{array}{l}\text { Otitis media }(N=50) \\
n(\%)\end{array}$ & $\begin{array}{l}\text { Otitis externa }(N=21) \\
n(\%)\end{array}$ & $\begin{array}{l}\text { All cases }(N=79) \\
n(\%)\end{array}$ \\
\hline Proteus mirabilis & $18(36)$ & $1(5)$ & $23(29)$ \\
\hline Staphylococcus aureus & $7(14)$ & $5(24)$ & $12(15)$ \\
\hline Pseudomonas aeruginosa & $7(14)$ & $7(33)$ & $16(20)$ \\
\hline Other bacteria ${ }^{*}$ & $4(8)$ & - & $6(8)$ \\
\hline Fungal & - & $1(5)$ & $1(1)$ \\
\hline Mixed growth/only skin flora ${ }^{\dagger}$ & $14(28)$ & $7(33)$ & $21(26)$ \\
\hline
\end{tabular}

true for all reports. Although some African studies reported $P$. mirabilis to be the most common organism, other international studies report $S$. aureus and $P$. aeruginosa to be the most common organisms encountered with chronic suppurative otitis media. ${ }^{[1,4-7]} \mathrm{A}$ recent Nigerian study reported that Gram-negative bacteria (Klebsiella spp., Escherichia coli and P. aeruginosa) comprised $80 \%$ of isolates. ${ }^{[]]}$ The microbiology with cholesteatoma is fairly similar, with a predominance of $P$. aeruginosa (31\%) and S. aureus (19\%), but there is also a significant anaerobic component. ${ }^{[8]}$

The incidence of MRSA in Cape Town is low compared with other studies. In our study, MRSA was isolated in only 1 patient (1.7\%) with otitis media for 2 years; it is not known what treatment the patient had received at primary care facilities. This is similar to the $1.9 \%$ incidence of MRSA reported by Loock ${ }^{[3]}$ in patients with chronic active otitis media. These results differ from reports of changing bacteriological spectra and increases in the incidences of S. aureus and MRSA. ${ }^{[1]}$ Hwang et al. ${ }^{[1]}$ reported from Taiwan that, when compared with Juan (1986), ${ }^{[1]}$ there had been an 8.5\% increase in the incidence of MRSA in acute otitis externa; they also reported an increase in MRSA with granular myringitis, and that the biggest change in microbiology profile - a 15\% increase in MRSA - had occurred with chronic otitis media. ${ }^{[1]}$ Similar trends were observed in a large study from Korea, with the prevalence of MRSA increasing from $21.4 \%$ to $27.4 \%$. ${ }^{[1]}$ Hwang et al ${ }^{[1]}$ postulated that the change from $P$. aeruginosa to $S$. aureus, especially MRSA, could be attributed to improvements in public healthcare, reduced severity of ear disease and a more liberal use of antimicrobial drug therapy.

At primary care level, chronic otorrhoea is treated medically with ear toilet according

Table 2. Antibiotic sensitivities of isolates from patients with otorrhoea for both otitis media and otitis externa

\begin{tabular}{|c|c|c|c|c|c|}
\hline & $\begin{array}{l}\text { P. mirabilis }(N=23) \\
n(\%)\end{array}$ & $\begin{array}{l}\text { P. aeruginosa }(N=16) \\
n(\%)\end{array}$ & $\begin{array}{l}\text { S. aureus }(N=12) \\
n(\%)\end{array}$ & $\begin{array}{l}\text { Other organisms }(N=6) \\
n\end{array}$ & $\begin{array}{l}\text { All organisms } \\
\text { (\%) }\end{array}$ \\
\hline Ciprofloxacin & $23(100)$ & $16(100)$ & $10(83)$ & 6 & 96 \\
\hline Gentamycin & $23(100)$ & $13(81)$ & $4(33)$ & 6 & 81 \\
\hline Amikacin & $23(100)$ & $16(100)$ & - & 5 & 77 \\
\hline Ampicillin & $20(87)$ & - & $1(8)$ & 2 & 40 \\
\hline $\begin{array}{l}\text { Amoxicillin/ } \\
\text { clavulanate }\end{array}$ & 23 & - & - & - & 41 \\
\hline Cefotaxime & 23 & - & - & - & 41 \\
\hline Cefuroxime & 22 & - & - & - & 39 \\
\hline Ceftazidime & - & 16 & - & 6 & 39 \\
\hline Co-trimoxazole & 14 & - & - & 4 & 32 \\
\hline Tobramycin & - & 10 & - & 6 & 28 \\
\hline Oxacillin & - & - & 11 & - & 19 \\
\hline Erythromycin & - & - & 11 & - & 19 \\
\hline
\end{tabular}




\begin{tabular}{ll} 
Table 3. Current SA DoH guideline for chronic otitis media & \\
\hline Non-drug treatment & $\begin{array}{l}\text { Avoid getting the inside of the ear wet } \\
\text { Dry mopping } \\
\text { Topical } 1 \% \text { acetic acid or diluted vinegar solution }\end{array}$ \\
& $\begin{array}{l}\text { Amoxicillin } \\
\text { Drug treatment }\end{array}$ \\
& $\begin{array}{l}\text { Penicillin allergy: Co-trimoxazole } \\
\text { SA = South African; DoH = Department of Health. }\end{array}$
\end{tabular}

to set protocols. Only when otorrhoea does not resolve are patients referred to a more specialised service. As otorrhoea generally is not cultured and an accurate distinction between otitis externa and media is usually not made at primary care level, commonly encountered pathogenic organisms should inform empiric treatment protocols. The current DoH guideline for treatment of chronic otitis media recommends amoxicillin or co-trimoxazole for patients allergic to penicillin (Table 3). ${ }^{[0]}$ Many of the patients in our study had received amoxicillin in accordance with this guideline. Although the majority of the $P$. mirabilis isolates were susceptible to amoxicillin, only $40 \%$ of organisms overall were susceptible.

The organisms isolated in our study were susceptible mainly to fluoroquinolones (96\%) and aminoglycosides (81\%) (Table 2). Topical fluoroquinolone eardrops, commonly ofloxacin or ciprofloxacin, are known to be as effective as aminoglycoside drops, and have the advantage of not being ototoxic. ${ }^{[10]}$ Ofloxacin has been shown to be very effective in all studies except for a study done in Malawi ${ }^{[6]}$ that reported that after an initial $73 \%$ response rate at 10 days, the response rate dropped to $42 \%$ at 21 weeks. However, most ears (in 91 candidates) in this study harboured faecal bacteria, and it was postulated that this finding was due not to bacterial resistance, but due to poor hygiene. ${ }^{[6]}$ In keeping with previous studies, Loock ${ }^{[3]}$ reported that topical quinolone eardrops were effective in the management of active chronic otitis media. Weber et al. ${ }^{[1]]}$ showed that no significant antibiotic resistance emerged from the use of topical antibiotics.

Our results suggest that should additional antibiotic therapy be considered in patients who have failed to respond to first-line therapy in a primary care setting, agents with better activity against Gram-negative bacilli should then be considered.

Another concern highlighted by our study was that $43 \%$ of patients had a discharging ear for $>52$ weeks. The reasons for this delayed referral to specialist centres require further research. We were unable to analyse the effect of HIV status on otorrhoea; further microbiological investigation at primary care level is required to determine whether it differs from HIV-negative patients.

\section{Conclusions}

Otorrhoea is generally treated at primary care level in SA and, hence, has to be protocol driven. Antimicrobials (topically or systemically) recommended for otorrhoea in the DoH guideline need to cover the majority of organisms encountered; be it due to otitis media, granular myringitis or otitis externa, as the primary healthcare worker is unlikely to distinguish between these causes of otorrhoea. The results of this study suggest that the microbial spectrum in the Western Cape, and possibly the rest of SA, differs from elsewhere in the world and that MRSA infection is uncommon.
Amoxicillin appears to be a suboptimal choice for chronic non-responders due to its low sensitivity when compared with topical fluoroquinolone eardrops with ear mopping, which covers $95 \%$ of organisms cultured and is not ototoxic. Should the first-line treatment fail then an antibiotic group with Gram-negative cover should be considered.

Acknowledgement. This study was made possible by funding from the SA Society of Otolaryngology Head and Neck Surgery.

\section{References}

1. Hwang JH, Chu CK, Liu TC. Changes in bacteriology of discharging ears. J Laryngol Otol 2002;116(9):686-689. [http:/ dx.doi.org/10.1258/002221502760237957]

2. Clinical Laboratory Standards Institute. Performance standards Clinical Laboratory Standards Institute. Performance standards
for antimicrobial susceptibility testing: 17th informational supplement. CLSI M100-S17;27(1). Wayne, PA: Clinical supplement. CLSI M100-S17;27(1). Wayne, PA: Clinical

Laboratory Standards Institute, 2007. http
bg.com/CLSI.pdf (accessed 14 May 2013)

3. Loock JW. A randomised controlled trial of active chronic otitis media comparing courses of eardrops versus one-of topical treatments suitable for primary, secondary and tertiary healthcare settings. Clin Otolaryngol 2012;37(4):261-270. [http://dx.doi.org/10.1111/j.1749-4486.2012.02532.x]

4. Yeo SG, Park DC, Hong SM, Cha CI, Kim MG. Bacteriology of chronic suppurative otitis media - a multi-center study. Acta Otolaryngol 2007;127(10):1062-1067. [http://dx.doi. org/10.1080/00016480601126978

5. Aslam MA, Ahmed Z, Azim R. Microbiology and drug sensitivity patterns of chronic suppurative otitis media. J Coll Physicians Surg Pak 2004;14(8):459-461.

6. van Hasselt $P$, van Kregten E. Treatment of chronic suppurative van Hasselt P, van Kregten E. Treatment of chronic suppurative
otitis media with ofloxacin in hydroxypropyl methylcellulose ear otitis media with ofloxacin in hydroxypropyl methylcellulose ea
drops: A clinical/bacteriological study in a rural area of Malawi. Int J Pediatr Otorhinolaryngol 2002;63(1):49-56. [http://dx.doi org/10.1016/S0165-5876(01)00648-6]

7. Adoga AA, Bakari A, Afolabi OA, Kodiya AM, Ahmad BM. Bacterial isolates in chronic suppurative otitis media: A changing pattern? Niger J Med 2011;20(1):96-98.

8. Ricciardeillo F, Cavaliere M, Mesolella M, Iengo M. Notes on the microbiology of cholesteatoma: Clinical findings and treatment. Acta Otorhinolaryngol Ital 2009;29(4):197-202.

9. South African Department of Health. The prevention of hearing impairment due to otitis media at clinic level. Pretoris hearing impairment due to otits media at clinc level. Pretoria: policy/2012/hearingguide.pdf (accessed 14 May 2013)

10. Manolidis S, Friedman R, Hannley M, et al. Comparative efficacy Manolidis S, Friedman R, Hannley M, et al. Comparative efficacy
of aminoglycoside versus fluoroquinolone topical antibiotic of aminoglycoside versus fluoroquinolone topical antibiotic
drops. Otolaryngol Head Neck Surg 2004;130(3):S83-S88. drops. Otolaryngol Head Neck Surg 2004,
[http://dx.doi.org/10.1016/j.otohns.2003.12.006]

[http://dx.doi.org/10.1016/j.otohns.2003.12.006]
Weber PC, Roland PS, Hannley M, et al. The developmen

11. Weber PC, Roland PS, Hannley M, et al. The developmen
of antibiotic resistant organisms with the use of ototopical medications. Otolaryngol Head Neck Surg 2004;130(3):S89-S94. [http://dx.doi.org/10.1016/j.otohns.2003.12.009]

Accepted 2 May 2013 